\title{
Photothermal beam deflection technique for non-destructive evaluation of thinfilm photovoltaic cells
}

\author{
Anita R Warrier ${ }^{1 *}$, K P Vijayakumar², C Sudha Kartha ${ }^{2}$, N Manoharan ${ }^{1}$, B Venkatraman ${ }^{3}$ \\ 1AMET University, Kanathur, Chennai-603103 \\ ${ }^{2}$ Cochin University of Science and Technology, Cochin-682022 \\ ${ }^{3}$ RSEG, IGCAR, Kalpakkam-603102 \\ ${ }^{\star}$ Corresponding author: anitawarrier2@gmail.com
}

\begin{abstract}
Photothermal beam deflection technique is used to measure the electronic transport properties of the photovoltaic cell layers at different fabrication stages. The open circuit voltage and short circuit current of the cell is shown to be depend on the transport properties of the window layer material. The photothermal technique is suitable adapted to measure the series resistance, optimum load resistance and photovoltaic efficiency of a three layer (transparent conducting oxide /absorber layer/window layer) compound semiconductor thin film photovoltaic cell. The cell parameter values measured using photothermal technique is in agreement with the electrically measured values. The possibility of efficient and continuous evaluation of the photovoltaic cell during different fabrication stages using photothermal technique is demonstrated.
\end{abstract}

\section{Introduction}

Real time characterization and continuous non-contact non-destructive evaluation of a device during its fabrication stage is important for improving the performance, quality and reliability of products in photovoltaic industry. A technique to measure the parameters that foretells any possible degradation and failure during the initial stages of fabrication is essential for lowering the production costs and to assuring the certified quality. Thermal wave techniques have gained increasing attention in process control mainly due to their non-invasive nature whereby any possibility of contamination can be avoided. Photothermal beam deflection technique (PTBD) is an efficient non-destructive method for evaluating semiconductor thin films and photovoltaic devices. Since demonstration for first time in 1880, these techniques have evolved in different stages and now do multi-purpose analyses of a variety of materials like polymers, ceramics, semiconductors and various biological samples [1]. Photothermal techniques are highly sensitive and have become a tool for absorption studies in both semiconductor films and devices [2]. This technique is shown to be effective for monitoring the device at processing stages and for rapidly identifying the changes during the processing itself [3]. The ability to monitor each stage of device processing can lead to the identification of parameters, which critically affect the device quality. This includes monitoring the quality of substrate, the subsequent device layers and determination of their electronic parameters.

Photothermal approach was adopted by Daniele Fournier et al [4] to measure transport processes based on the principle of optical beam deflection. This measurement technique, which is an extension of photothermal deflection, has significant advantages as it is contactless and directly yields both thermal and electronic transport parameters within the bulk / at surface / interface of a semiconductor. Another important advantage of the technique is that it can be used to measure these transport properties in a spatially resolved $\left(\mu^{3}\right)$ manner. This model allows for the explicit measurement of important parameters such as thermal and electronic diffusivity, electronic mobility, and carrier recombination kinetics-both in bulk and surface or interface. Later, in 1995, photoacoustic (PA) heat-transmission measurements were used to study transport in a nearly intrinsic Ge single crystal by M D Dramicanin et al [5]. D M Todorovic et al [6] investigated photoacoustic effect as a function of the modulation frequency in a 'transmission-detection configuration' for semiconductor samples. Dependence of photoacoustic effect on thermal diffusion, thermoelastic, and electronic-transport parameters was identified. Infrared photothermal radiometry was found to be extremely useful in measuring thermal and several electronic properties of semiconductors. Photothermal radiometry (PTR) allows measurement of optically induced black body radiation from semiconductor surface. It has been shown that the frequency-domain PTR signal is extremely sensitive to the photoexcited carrier plasma-wave in semiconductors. Hence this technique has been attractive for ion implantation and process monitoring with carrier plasma wave technology. Applications of technique to ion implanted Si wafer for qualitative analysis of the influence of thermal annealing and sensitivity of the carrier plasma wave to implantation induced damage in Si technique have been reported [7]. This technique was successfully demonstrated to be capable of characterization of ion implantation effects on the electronic properties, as well as monitoring the contamination of Si wafers [8] and Si electronic devices [9]. But all these works were done under the assumption that semiconductors were homogeneous or discontinuously homogeneous; however modern device processing requires improvement of the spatial resolution of PTR, allowing for measurements within the 'scribe lines'. Three dimensional measurement techniques taking into 
account of the finite size of excited laser beam, the sample thickness and plasma-thermal wave contributions to the signal were adopted by Tetsuo lkari et al [10] for analysing semiconductor wafers patterned for LSI circuits. Photothermal deflection technique was used for measuring thermal diffusivity of double epitaxial layer of n-type GaAs doped with various concentrations of $\mathrm{Si}$ and a p-type Be doped $\mathrm{GaAs}$ layer grown on $\mathrm{GaAs}$ substrate by molecular beam epitaxial method. Thermal diffusivity is evaluated from the slope of the graph of the phase of the photothermal signal as a function of pump-probe offset. Doping concentration was found to have a great influence on the thermal diffusivity value [11].

Studies using photopyroelectric technique have shown that the generation and propagation of thermal waves in crystalline $\mathrm{Si}$ depends on the thermal history and formation native a- $\mathrm{SiO}_{2}$ in the sample. It was also used for analysis of recombination processes in semiconductors [12]. Photothermal techniques were also used for measuring the optical and thermal properties of porous silicon [13]. This technique also finds application in measuring thermal and electronic transport properties of CdTe thin films and powders of RuTe, RuS, RuSe $e_{2}$ and CdTe [14]. E Marin et al determined the non-radiative lifetime in direct band gap semiconductors, such as GaAs, GaSb and InSb [15]. A Mandelis et al showed that a new photothermal technique of 'lock-in-rate window infrared radiometery' is capable of completely separating out photoexcited free-carrier-wave and thermal wave contribution to the photothermal signal from an n-type, Cr-doped Si wafer with a superior temporal resolution in the determination of electronic lifetime and thermal transport time constant [16]. Three dimensional photothermal radiometric microscopic imaging and laser-intensity-modulation frequency scan were used for the non-contact measurements of electronic transport properties of integrated circuits in patterned Si wafers [17]. Laser induced infrared photocarrier radiometry is a powerful tool for carrier density diagnosis through non-contact characterization in semiconductors. It was employed to determine the temperature dependence of carrier mobility in Si wafers [18].

David Cahen et al (1978) used PA measurements to characterize photovoltaic devices. Such measurements are useful for the optimisation of multicomponent, photovoltaic systems such as semiconductor/liquid junction devices [19]. Later in 1984, yet another work by same group showed PA phase angle dependence on series resistance and how phase shift can be used for interpretation of thermal losses in PV cells [20]. The photothermal pyroelectric technique is applied for the characterization of photovoltaic cells and they have compared results with the photoacoustic detection using a simple model for junction type solar cell [21]. S I Yun used PA technique for measuring energy conversion efficiency and quantum efficiency of photovoltaic process in Si solar cell [22]. Reitte et al have reported on the additional thermal losses in PV signal of Si solar cells due to current dissipation in the internal resistance of the cell $[23,24]$. P Grunow et al [25] used photothermal deflection to investigate electronic transport in silicon solar cells. They simulated the dependence of deflection signal on external bias voltage in the closed circuit condition and additional bias illumination on open circuit condition. Diode parameters describing the dark currents, due to recombination at interface or in the space charge region, are used, as free parameters to fit experimental data and the values were identical with conventional electrical measurements. This technique can be used for contactless quality control of silicon solar cells in different stages of their preparation process. Photoacoustics was used as calorimetric method in conjunction with electrical measurements to determine the mechanisms involved in conversion of most of the absorbed radiation to thermal energy in solar cells. Harvey Flaisher et al formulated a model for heat generation in solar cells that allowed quantitative separation of loss mechanisms. These results were proven for Si solar cells and CulnSe $/ \mathrm{CdS}$ thin film solar cell [26]. Karem Boubaker developed an enhanced photothermal technical protocol for diagnosing spatial distribution of dislocation inside photovoltaic polycrystalline silicon solar cells [27]. In this work we focus on the development of the photothermal beam deflection technique for investigating the opto-electronic properties of semiconductor thin films and also determination of cell parameters for optimization of cell fabrication conditions. This application can be used for quality control of solar cell in different stages of their deposition process. In the first step the transport parameters, thermal diffusivity $\left(D_{t h}\right)$, Carrier mobility $(\mu)$, surface recombination velocity $(V s r)$ and carrier life time $\left(\tau_{r}\right)$ can be determined to check quality and finally junction parameters can be determined using this technique.

\section{Theoretical background}

Transverse PTBD technique, in skimming configuration, where the probe beam propagates perpendicular to the pump beam and at a fixed distance from the sample surface, has been widely studied by several groups [28-31] since past three decades. Primary source of photothermal signal arises due to the periodic heat flow from solid to its surrounding as the solid is heated using a modulated light. Only a relatively thin layer of medium $(0.2 \mathrm{~cm}$ for a chopping rate of $100 \mathrm{~Hz}$ ) adjacent to the surface of the solid responds thermally to the periodic heat flow from the solid. This is a diffusive process that produces a periodic temperature distribution and the amplitude of the signal thus generated depends on the quantity of the heat emanating from the illuminated sample; there is a close correspondence between the strength of the signal and the amount of light absorbed. Photothermal signal amplitude also depends on the sample's overall light absorption irrespective of whether it is volume or surface related. On other hand, photothermal phase signal depends also on the localization of the absorption. In fact, in an absorbing sample, the light absorbed in part or in the entire sample volume acts as an effective centre of heating source, whose position depends on the sample optical absorption depth of the sample and thermal 
diffusion length. Photothermal beam deflection theory shows that the transverse deflection from the original trajectory of the probe beam depends on the thermal gradients in the direction orthogonal to the path only according to the well-known formula, which, in geometric optics, is written as,

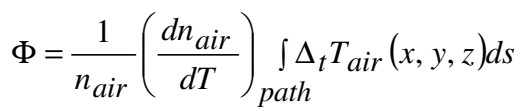

Where $\mathrm{n}_{\text {air }}, \mathrm{dn}_{\mathrm{air}} / \mathrm{dT}$ represent the air refractive index and the optothermal parameter respectively, $\mathrm{T}_{\text {air }}$ is the temperature rise in air, and $\Delta_{\mathrm{t}}$ is the gradient transverse to the probe paths.

A one-dimensional model of heat flow in the cell resulting from the absorbed light energy was put forth by Rosencwaig et al [32] for measuring the signal generated in photoacoustic technique. Later in 1990, U Zammit et al [33] developed a 1-D theoretical model for analysis of photothermal deflection signal amplitude and phase. According to this theory any light absorbed by the solid is converted, in part or whole, into heat by non-radiative deexcitation processes within the solid. When a semiconductor material is illuminated with photons of sufficient energy, electron-hole pairs are generated which diffuse through the crystal from their place of generation to regions of lower excess pair concentration. Each pair transports energy approximately equal to the band separation, thus contributing to the thermal conductivity. This energy is deposited where the pair undergoes nonradiative recombination increasing the local measurable temperature of the lattice. A non-uniform temperature distribution is therefore established in the sample which depends on the nature of the incident radiation, on the characteristics of optical absorption, on the boundary conditions for temperature and energy flux, and on the ordinary thermal conductivity of the material.

\subsection{One-dimensional theoretical model for photothermal wave generation and propagation in semiconductor thin films}

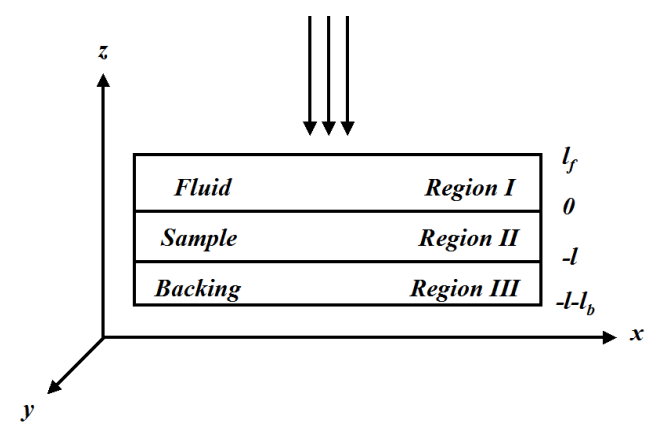

Fig 1 Three-layer sample geometry (backing-sample-fluid)

Theoretical model for photothermal analysis of semiconductor put forth by $U$ Zammit et al [33] does not include non-radiative recombination and surface recombination processes taking place in the semiconductors and the model given by Fournier et al [34] does not suit the three layer sample geometry with fluid, sample and backing layer; hence the present model was extended to suit the transverse photothermal beam deflection technique with three-layer sample geometry, assuming the sample to be a semiconductor thin film. Accuracy of the measurement technique can be improved by further refinement of the theoretical model. We applied this technique for simultaneous measurement of minority carrier lifetime $\left(\tau_{r}\right)$, surface recombination velocity $\left(V_{s r}\right)$ and thermal diffusivity $\left(D_{t h}\right)$ of semiconductor thin films. This technique has further scope for improvement as it can be extended into large area applications as well.

Let us consider the three-layer sample model geometry as shown in figure 1. The sample under investigation is irradiated with an intensity modulated laser beam with angular frequency $\omega_{\bmod }=2 \pi f$, where $f$ is the modulation frequency. Intensity of the laser beam is given by,

$I=\frac{I_{0}}{2} e^{-\beta z} \operatorname{Re}\left[1+e^{i \omega t}\right]$ 
Where $l_{0}$ is the incident monochromatic light flux $\left(\mathrm{W} / \mathrm{m}^{2}\right), \beta$ is the absorption coefficient $\left(\mathrm{m}^{-1}\right)$ of the illuminated sample and $t$ is the time. It is assumed that the excitation beam energy $\mathrm{E}=\mathrm{hv}$, is greater than the band gap of the material, where ' $h$ ' is Planck's constant and ' $v$ ' is the frequency of the beam.

The semiconductor is considered to be isotropic, thermally and electronically, and we assume that fluid and backing do not absorb the incident beam and hence they donot act as thermal sources. This is true in this case as we used light beam from a He-Ne laser for the optical excitation. The sample is assumed to be optically thin $\left(\mu_{\beta}>I\right)$ and thermally thin $\left(\mu_{s}>I\right)$, where $\mu_{\beta}$ and $\mu_{s}$ is optical penetration length and thermal diffusion length of the sample respectively. Hence the optical absorption is taken to be finite i.e., the absorption takes place throughout the sample volume rather than sample surface alone. Excitation beam is assumed to be of finite size with guassian profile, having spot size larger than the electronic diffusion length of the material. Hence a onedimensional treatment of the heat diffusion equations is sufficient.

\subsection{Heat diffusion equations}

The light absorbed by a sample is converted by part or whole into heat by non-radiative deexcitation processes in the material. Thermal conduction is a diffusive process by which the generated heat is transferred from point of origin to other parts of the sample and its surroundings as a result of temperature gradient. Heat conduction is a diffusive process governed by a parabolic differential equation, which lacks second derivative with respect to time. From Fourier's law for heat conduction, the heat flux is proportional to negative of local temperature gradient.

$q=-k A \frac{d T(z, t)}{d z}$

The continuity equation is given by,

$\rho c \frac{d T(z, t)}{d t}=-\frac{d q}{d z}$

Where $k$ is the thermal conductivity $(\mathrm{W} / \mathrm{mK}), T(z, t)$ is the temperature, $\rho$ is the density $\left(\mathrm{kg} / \mathrm{m}^{3}\right)$ and $c$ is the specific heat $(\mathrm{J} / \mathrm{kg} \mathrm{K})$, space coordinate $z$ and time $t$.

The heat diffusion equation in one-dimensional form, when there is no heat source and sink is given by,

$$
\frac{\partial T(z, t)}{\partial t}=\frac{1}{D_{s}} \frac{\partial^{2} T(z, t)}{\partial z^{2}}
$$

Where $T(z, t)$ is the temperature, $D_{s}$ is the thermal diffusivity given by $k / \rho c\left(\frac{c m^{2}}{s}\right)$

A semiconductor thin film absorbs the photons with energy greater than the band gap [ $\left.E_{g}\right]$ of the material, resulting in excess carrier distribution within the semiconductor with energy above (below) the conduction (valence) band. In a time scale of few pico seconds, these photo-injected carriers distribute the energy within themselves through coulomb interaction and finally this extra energy is given to lattice by relaxing to the bottom of the conduction band via carrier phonon interaction. As the excess carriers diffuse through the sample the electron hole pairs will eventually recombine producing a second source of heat. This will also diffuse into the semiconductor. The heat diffusion equation including the heat sources is given by,

$$
\frac{\partial T(z, t)}{\partial t}=\frac{1}{D_{s}} \frac{\partial^{2} T(z, t)}{\partial z^{2}}+Q(z, t)
$$

The three major heat sources are defined by $Q(z, t)$, the net heat power density, are

2.2.1. Nonradiative intraband thermalization: When photo generated carriers relax down to the bottom of the conduction band by creating phonons, the heat power density is given by, 
$Q_{T}(z, t)=\beta \frac{\left(h v-E_{g}\right)}{h v} I_{0} e^{-\beta z\left(1+e^{i \omega t}\right)}$

2.2.2. Nonradiative bulk recombination: Non-radiative recombination of excess electron hole pairs after diffusion occurs through a distance $\left({ } D_{n} \tau_{r}\right)$ where $D_{n}$ is the carrier diffusivity $\left(\mathrm{m}^{2} / \mathrm{s}\right)$ and $\tau_{\mathrm{r}}(\mathrm{s})$ is the carrier lifetime.

The heat power density due to non-radiative recombination is given by,

$Q_{N R}=\frac{E_{g}}{\tau_{r}} N(z, t)$

Where $N(z, t)$ is the density of photo carriers, $\tau_{r}$ is the nonradiative recombination time.

Therefore the total power density is given by,

$Q=Q_{T}+Q_{N R}$

2.2.3 Nonradiative surface recombination: When nonradiative recombination takes place at surface, a heat power density is,

$Q_{S R}=E_{g} V_{s r} N(z, t)$

This thermal contribution has to be included in the boundary condition of heat diffusion equations.

\subsection{Minority carrier diffusion equation}

When a beam of light irradiates the sample of thickness $L$, it leads to photo carrier generation; the excess carriers generated can be calculated from minority carrier diffusion.

The continuity equation can be written as,

$$
\frac{d n}{d t}=\left.\frac{d N}{d t}\right|_{\text {diffusion }}+\left.\frac{d N}{d t}\right|_{\text {drift }}+\left.\frac{d N}{d t}\right|_{\text {rec-gen }}+\left.\frac{d N}{d t}\right|_{\text {other process like light.... }}
$$

Where ' $N$ ' is the minority carrier concentration. Hence the drift term can be omitted as we consider only low-level injection.

$\frac{d n}{d t}=\left.\frac{d N}{d t}\right|_{\text {diffusion }}+\left.\frac{d N}{d t}\right|_{\text {rec-gen }}+\left.\frac{d N}{d t}\right|_{\text {other process like light.... }}$

For photo-generated carriers the transport equation is given by,

$$
\frac{d N(z, t)}{d t}=D_{n} \frac{d^{2} N(z, t)}{d z^{2}}-\frac{\Delta N}{\tau_{r}}+G(z, t)
$$

Where $G(z, t)$ is the generation rate of the carriers,

$$
G(z, t)=\frac{\beta I_{0}}{2 h v} e^{-\beta z}
$$

To evaluate the excess charge carrier distribution, we make the following assumptions:

i) When the material is in steady state $\frac{d N(z, t)}{d t}=0$ 


\section{http://dx.doi.org/10.21611/qirt.2015.0136}

ii) Since absorption takes place throughout the material thickness, excess carriers are created throughout the material and there is no carrier diffusion along the $z$ direction.

Hence the minority carrier diffusion equation can be written as,

$$
\begin{aligned}
& \frac{\Delta N}{\tau_{r}}=G(z, t) \\
& \frac{\Delta N}{\tau_{r}}=\frac{\beta I_{0}}{2 h v} e^{-\beta z}
\end{aligned}
$$

The heat diffusion equation in the three regions, fluid, sample and backing is,

$$
\begin{aligned}
& \frac{\partial^{2} T_{f}(z, t)}{\partial z^{2}}=\frac{1}{D_{f}} \frac{\partial T_{f}}{\partial t} \\
& \frac{\partial^{2} T_{s}(z, t)}{\partial z^{2}}=\frac{1}{D_{s}} \frac{\partial T_{s}}{\partial t}-Q_{T}-Q_{N R}
\end{aligned}
$$

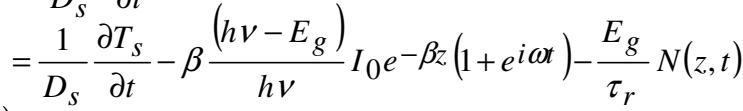

$$
\begin{aligned}
& \frac{\partial^{2} T_{b}(z, t)}{\partial z^{2}}=\frac{1}{D_{b}} \frac{\partial T_{b}}{\partial t}
\end{aligned}
$$

One of the boundary conditions is the continuity of temperature at the region boundaries,

$$
\begin{aligned}
& \left.T_{f}(z, t)\right|_{z=0}=\left.T_{s}(z, t)\right|_{z=0} \\
& \left.T_{s}(z, t)\right|_{z=-l}=\left.T_{b}(z, t)\right|_{z=-l} \\
& \left.T_{f}(z, t)\right|_{z=+\infty}=\left.T_{b}(z, t)\right|_{z=-\infty}
\end{aligned}
$$

The next boundary condition is obtained from the heat continuity equation, which states that the heat flux flowing out of one region must be equal to heat entering into the adjoining region, must be obeyed.

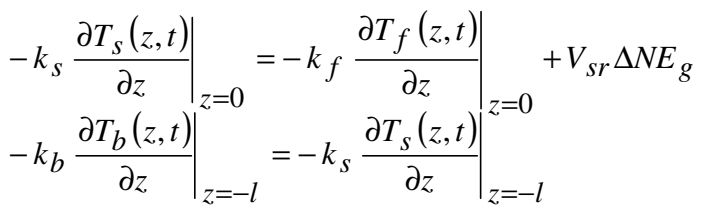

Where $k_{i}, D_{i}$ with $i=f, s, b$, represents the thermal conductivity and thermal diffusivity of fluid $(f)$, sample $(s)$ and backing $(b)$ respectively.

On solving the thermal diffusion equation (16) with boundary conditions (17) and (18), we get the following expression for complex amplitude of temperature $T(z, t)$ in the three regions.

$$
\begin{aligned}
& T_{f}(z)=T_{s} e^{-\sigma_{f} z} \\
& T_{s}(z)=C_{1} e^{-\sigma_{s} z}+C_{2} e^{-\sigma_{s} z}-\left(C_{3}+C_{4}\right) e^{-\sigma_{s} z} \\
& T_{b}(z)=W e e_{b}(z+l)
\end{aligned}
$$

Where, 


$$
\begin{aligned}
C_{3} & =\frac{\beta I_{0}}{2 k_{s}}\left(\frac{E-E_{0}}{E}\right) e^{-\beta z} \\
C_{4} & =\frac{E_{g}}{\tau_{r} k_{s}} \frac{\beta I_{0}}{2 h v} e^{-\beta z} \\
\sigma_{i} & =\sqrt{\frac{i \omega}{D_{i}}}
\end{aligned}
$$

The coefficients $C_{1}, C_{2}$ and $W$ were obtained using the boundary conditions. Using $C_{1}, C_{2}, C_{3}$, and $C_{4}$ in equation 19 , we finally obtain the surface temperature distribution is given by the equation 21 .

$$
\begin{aligned}
& T_{s}=\left\{\frac{\left[\begin{array}{l}
\sqrt{D_{f} D_{s}}\left(-R_{1} B \sigma_{b}^{2} D_{b}^{\frac{3}{2}} k_{s}+R_{2} D_{s}\left\{\begin{array}{l}
\beta \sqrt{D_{s} k_{b}}\left\langle B \beta+C k_{s}\right\rangle- \\
\sigma_{s}\left\langle B \sigma_{b} \sqrt{D_{b} k_{b}}-C \sqrt{D_{b} k_{s}^{2}}\right\rangle
\end{array}\right\}\right) \\
D_{s} k_{s}\left(R_{3} C\left\{\sigma_{s} \sqrt{D_{s} k_{b}}+k_{s} \beta \sqrt{D_{b}}\right\}+R_{1} B \beta^{2} \sqrt{D_{b}}\right)
\end{array}\right]}{\left[\sqrt{i \omega}\left(i \omega-\beta^{2} D_{s}\right)\right.}\left\{\begin{array}{l}
R_{2}\left\{D_{s} k_{b} k_{f}+\sqrt{D_{b} D_{f}} k_{s}^{2}\right\}+ \\
\left.\left.R_{1}\left\{\sqrt{D_{s} k_{s}}\left(\sqrt{D_{f} K_{b}}+\sqrt{D_{b} k_{f}}\right)\right\}\right)\right]
\end{array}\right\}\right. \\
& \text { where } \\
& R_{1} \rightarrow 1+e^{2 l \sigma_{s}} \\
& R_{2} \rightarrow-1+e^{2 l \sigma_{s}} \\
& R_{3} \rightarrow R_{1}-2 e^{l\left(\beta+\sigma_{s}\right)} \\
& B \rightarrow V_{s r} N(0) E_{g} \\
& C \rightarrow C_{3}+C_{4}
\end{aligned}
$$

\subsection{Photothermal beam deflection signal}

Refractive index gradient in the medium, surrounding the sample, due to heat generated from the sample is given by,

$$
\frac{d n}{d z}=\frac{d n}{d T} \frac{d T(z, t)}{d z}
$$

The deflection of probe beam (signal) thus produced is proportional to the refractive index gradient,

$$
\text { Signal } \alpha \frac{1}{n_{0}} \frac{d n}{d T} \frac{d T(z, t)}{d z}
$$

\subsection{Determination of thermal diffusivity, minority carrier lifetime and surface recombination velocity}

The parameters thermal diffusivity $\left(D_{s}\right)$, minority carrier lifetime $\left(\tau_{r}\right)$ and surface recombination velocity $\left(V_{s r}\right)$ are to be obtained as 'variable multiple fit parameters' by fitting the experimentally and theoretically obtained plot of modulation frequency versus signal amplitude (Figure 2). The experiment was performed first on silicon wafer and there was good agreement with the experimental data. Transport parameters $D_{s}, \tau_{r}, V_{s r}$ thus obtained were matching with earlier reported values. 


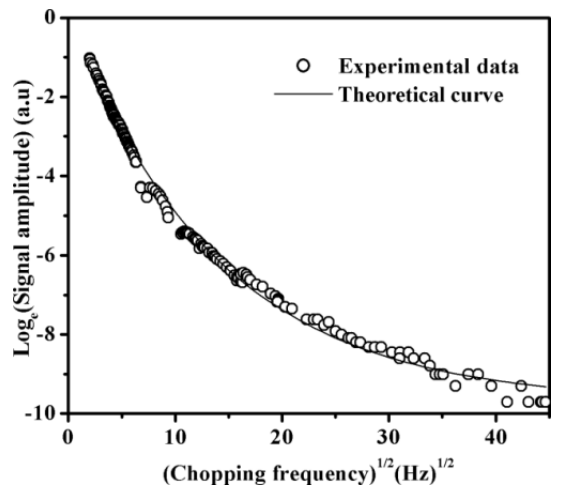

Fig 2 Experimental and theoretical fit for semiconductor thinfilm

\subsection{Measurement of electrical properties of photovoltaic cell by photothermal technique}

When a photon with energy higher than the bandgap irradiates a photovoltaic device, it will be absorbed and converted into useful electricity or converted into heat or light. We can measure the useful electrical output of the device by current and voltage analysis and also by photocurrent spectroscopy. The part of the absorbed light energy which is released in the form of heat add a definite contribution to the photothermal signal detection. Thus, the photothermal signal is proportional to the light into heat conversion efficiency and complementary to all other photoinduced energy-conversion processes. Apart from the non-radiative de-excitation processes, heating due to internal thermal loss in the cell also produces photothermal signal. This was identified as due to the current dissipation at the solar cell internal resistance [35].

In general form, photothermal signal may be written as

Signal $\propto \alpha\left[1-\sum\left(\phi_{i} \Delta E_{p, i} / N h v\right)-\left(\Phi_{l} v_{l} / v\right)-\gamma\right]$

where $\alpha$ is the fraction of light absorbed by the illuminated sample, $\Phi_{i}$ is the quantum yield for $\mathrm{i}^{\text {th }}$ photochemical reaction, $\Delta E_{\mathrm{p}, \mathrm{i}}$ is the molar internal change of product information in this reaction, $\mathrm{v} / \mathrm{and} \mathrm{v}$ are frequencies of emitted and incoming radiation, $N$ is the Avagadro number, $h$ is Planck's constant, $\gamma$ is the energy-conversion efficiency for photovoltaic process.

In a solar cell under photothermal analysis if only a photovoltaic process takes place the signal can be written as

Signal $\propto \alpha[1-\gamma(L)]$

where $L$ is the specific load resistance

Here, $[1-\gamma(L)]$ can be termed as the "photovoltaic loss" as it represents the decrease in the photothermal signal with respect to the normal absorption signal, because of photovoltaic process. From Eq 25, the photothermal conversion efficiency is given by

$\gamma(L)=[\alpha-\operatorname{Signal}(L)] / \alpha$

By minimizing the photothermal signal (i.e., by maximizing the term $(\alpha-\operatorname{signal}(L)$, we can find the optimum load resistance $(L)$ and conversion efficiency $(\gamma)$ at this load. As a photovoltaic device will have zero-energyconversion efficiency at an open circuit $(O C)$ condition, the photothermal signal (signal (OC)) under these conditions will be equal to $\alpha$, and therefore,

$\gamma(L)=\left[\operatorname{signal}(O C)-\operatorname{signal}\left(R_{L}\right)\right] / \operatorname{signal}(O C)$ 
Using equation 27, we can calculate the conversion efficiency of the photovoltaic device at a given $R_{L}$ from the ratio of the photothermal signal amplitude under the optimal load (signal $\left.\left(R_{L}\right)\right)$ and open circuit conditions (signal $(O C))$.

According to the maximum power transfer theorem, if $R_{L}$ is made larger than $R_{S}$, then the efficiency is higher, since a higher percentage of the source power is transferred to the load, but the magnitude of the load power is lower since the total circuit resistance goes up. If $R_{L}$ is smaller than $R_{S}$, then most of the power ends up being dissipated in the source, and although the total power dissipated is higher, due to a lower total resistance, it turns out that the amount dissipated at the load is reduced and $\gamma$ becomes negative for $R_{\mathrm{L}}<<R_{\mathrm{S}}$. Furthermore, at the matching resistance condition $R_{\mathrm{L}}=R_{\mathrm{S}}$ it predicts $\mathrm{Y}=0$. Therefore, from the plot of $\gamma\left(R_{\mathrm{L}}\right)$ versus $R_{\mathrm{L}}$, we can determine $R_{\mathrm{S}}$ (using the condition when $R_{\mathrm{L}}=R_{\mathrm{S}}, \mathrm{Y}=0$ ) and optimum $R_{\mathrm{L}}$ where the $\gamma$ is maximum.

\section{Experimental details}

\subsection{Photothermal beam deflection setup for evaluation of semiconductor thin films}

The beam from the He Ne laser of wavelength $632 \mathrm{~nm}$ [10 mW] with a spot size of $1 \mathrm{~mm}$ is focused to a diameter of $100 \mu \mathrm{m}$ using a convex lens and directed to the center of the sample using mirrors. The sample cell is a cylindrical crucible fixed on a XYZ translation stage and is provided with three windows for passing the pump, probe, and deflected beam. The sample is mounted vertically on the sample holder and immersed into the crucible containing $\mathrm{CCL}_{4}$. By placing the sample in the holder, the noise due to vibrations in the room can be reduced. $\mathrm{CCl}_{4}$ was chosen as the coupling medium since it absorbs only at a wavelength less than $250 \mathrm{~nm}$ and has a high temperature coefficient of refractive index $\left(5 \times 10^{-4} \mathrm{~K}^{-1}\right)$ nearly $10^{2}$ times higher than for air $\left(1 \times 10^{-6} \mathrm{~K}^{-1}\right)$. The probe beam $(546.5 \mathrm{~nm}, 1 \mathrm{~mW})$ is allowed to graze parallel to the sample surface at a distance of $100 \mu \mathrm{m}$ and perpendicular to the pump beam axis. The backing material [the substrate] and fluid are not light absorbing; hence they do not contribute to heat generation. Only a relatively thin region of fluid surrounding the sample adjacent to the surface responds thermally to the periodic heat flow from the film to the surrounding fluid. The deflection in the path of the probe beam is detected using the Bi-cell position sensitive detector (PSD) (EG \&G, UV-140, BQ-2). The entire setup is placed on an optical breadboard placed on a vibration isolation table, and thus the noise could be reduced to a certain extent. The signal is then amplified and recorded using a dual phase lock- in amplifier (SR830) for making sensitive and accurate measurements. The experimental setup is interfaced with the computer using Lab View 7.1 via an RS232 serial port for data acquisition and system control. A schematic sketch of the entire experimental setup is shown in Figure 3. In the present work also for determining the transport properties of CulnS $S_{2}$ and $\ln _{2} S_{3}$ layers that are used as an absorber layer and window layer in solar cells, the PTBD technique with the same configuration is used. However, the wavelengths of the beam used for the absorber layer $\left(C_{1} \mathrm{InS}_{2}\right)$ and window layer $\left(\beta-\mathrm{In}_{2} S_{3}\right)$ are $632 \mathrm{~nm}$ and $442 \mathrm{~nm}$ respectively depending on the bandgap energy of the investigated material. The diameter of the beam is kept at $100 \mu \mathrm{m}$ for both wavelengths.

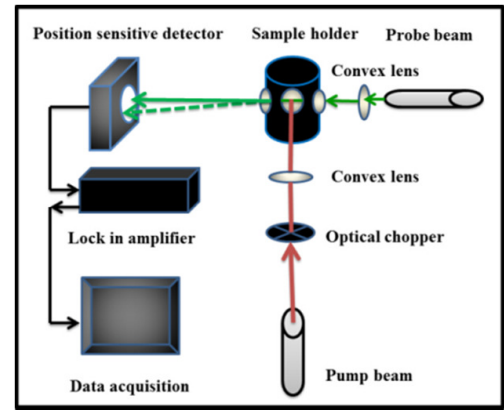

Fig 3 Schematic sketch of experimental arrangement of photothermal beam deflection technique

\subsection{Photothermal beam deflection techniques for evaluation of photovoltaic cells}

The photovoltaic device $\left(1 \times 1 \mathrm{~cm}^{2}\right)$ to be analysed is placed inside the sample cell, which is a cylindrical crucible with three windows. A schematic sketch of the experimental setup is shown in Figure 4. The experiment is done in an open circuit mode. In the open circuit mode, the constant bias illumination is supplied by a $250 \mathrm{~W}$ halogen lamp filtered with an IR filter. The load resistance $\left(R_{\mathrm{L}}\right)$ is connected to the solar cell device using external wires that are attached to the electrodes of the device. The material is excited with an intensity modulated pump beam $(2.81 \mathrm{eV})$ with a diameter of $100 \mu \mathrm{m}$ at a frequency of $15 \mathrm{~Hz}$. 


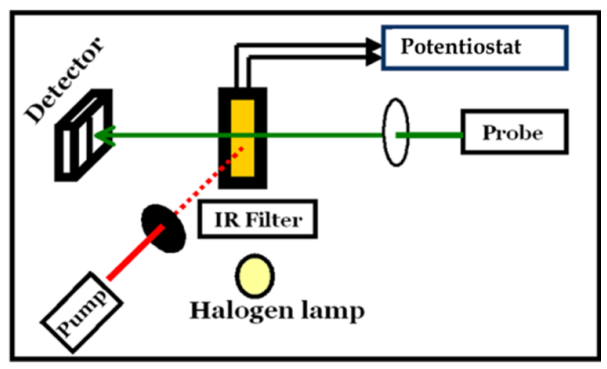

Fig 4 Photothermal experimental design for determination of electrical properties of photovoltaic cell

\subsection{Fabrication of Culn $\mathrm{S}_{2} / \ln _{2} \mathrm{~S}_{3} / \mathrm{Ag}$ Heterojunction}

The photovoltaic cell investigated in this work consists of three layers, the substrate (Indium tin oxide (ITO) coated over glass slides), the absorber layer and the window layer. The $\mathrm{p}-\mathrm{n}$ junction photovoltaic cell consisting of CulnS $S_{2}$ as absorber layer and $\beta$ - $\ln _{2} S_{3}$ as window layer is fabricated by chemical spray pyrolysis technique. For optimization of the composition of the films we measure the transport properties of $\mathrm{CulnS}_{2}$ thinfilms with three compositions (ie. $\mathrm{Cu} / \mathrm{In}$ is varied as $0.5,1$ and 1.5). Similarly the composition $\beta$ - $\ln _{2} \mathrm{~S}_{3}$ film is optimized by determining the transport properties of the film with varied $\operatorname{In} / \mathrm{S}$ ratio $(1.2 / 8,2 / 8,2.2 / 8,2.5 / 8,2.8 / 8$ and $3 / 8)$. The photovoltaic cell is fabricated by coating CulnS $\mathrm{S}_{2}$ layer over ITO coated glass slides. $\beta$ - $\ln _{2} \mathrm{~S}_{3}$ layer is coated over the CulnS $S_{2}$ layer and finally $\mathrm{Ag}$ layer is coated over the $\beta-\ln _{2} \mathrm{~S}_{3}$ layer functions as the electrode. The thickness each layer is $~ 100 \mathrm{~nm}$.

\section{Optimization of composition of CulnS $_{2}$ thin films}

Thin films of CulnS 2 were excited using the intensity-modulated beam of wavelength $632 \mathrm{~nm}$ and the deflection of probe beam was detected using a bi-cell PSD. A Plot of Log (Signal amplitude) versus (chopping frequency) ${ }^{1 / 2}$ (Figure 5) clearly shows dependence of signal on frequency. The general trend observed for CulnS $_{2}$ films, irrespective of the fabrication condition, is the rapid decrease in signal with frequency in the low frequency regime $(\leq 100 \mathrm{~Hz})$ and gradual or more "slow" decrease in the high frequency regime $(\geq 100 \mathrm{~Hz})$. Nature of dependence of signal amplitude on the chopping frequency and the frequency from which the change of slope (showing the 'rapid' or 'slow' decrease) alters with changes in the composition and surface morphology of the investigated film assist us in finding out the origin of non-radiative processes in the film. We have made a study on the impact of alternations in film composition on the transport properties of film. This can be correlated to the fabrication process and hence useful for optimisation of fabrication process.

Concentration of copper and indium in the spray solution has to be controlled. The excess or deficiency of the "metal" ( $\mathrm{Cu} \& \mathrm{In})$ in the film would alter the film's properties. So it is essential to choose the concentration of constituents in the film in accordance with device requirements. For the present study, films were prepared by varying the ratio $\mathrm{Cu} / \mathrm{In}$ as $0.5,1$ and 1.5 , and figure 5 shows variations in the photothermal signal plot for different $\mathrm{Cu} / \mathrm{In}$ in the film. We observed that for $\mathrm{Cu}$ deficient samples $(\mathrm{Cu} / \mathrm{In} \sim 0.5)$ the decrease in signal amplitude with modulation frequency is almost linear in the low frequency regime while in high frequency region, the signal decrease is very slow (Figure 5). In other samples, the contribution from defects in the bulk is high in the low frequency region. As a result, we could not observe a sudden or linear decrease in signal amplitude. Instead, there is a slow variation in which the thermal contribution is due to immediate thermalization of carriers to the bottom of conduction band and bulk recombination with release of phonons. Transport properties determined from the theoretical best-fit show that $V_{s r}$ is very high for $\mathrm{Cu}$ deficient samples, while for $\mathrm{Cu}$ rich $(\mathrm{Cu} / \mathrm{In} \sim 1.5)$ samples, $V_{s r}$ reduced by $10^{4}$ times. These values were in good agreement with reported values [36, 37]. The crystallinity and surface morphology is better for Cu rich films. Surface quality of Cu-rich films has improved due to better crystallinity mainly induced by the $\mathrm{Cu}_{2-x} \mathrm{~S}$ phase. Cu deficiency in a sample surface can cause formation of complex defects in the surface. For stoichiometric films $(\mathrm{Cu} / \mathrm{ln} \sim 1)$, carrier lifetime was the highest and mobility was also higher compared to $\mathrm{Cu}$ rich and $\mathrm{Cu}$ poor films (Table 1). Hence the stoichiometric films are best suited for device fabrication. 


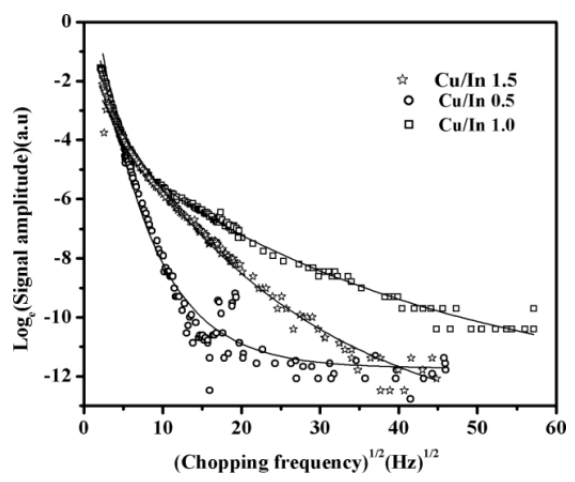

Fig 5 Photothermal responses of CulnS ${ }_{2}$ films with Cu/ln $0.5,1,1.5$

Table 1 Fit data obtained for CulnS ${ }_{2}$ thin films prepared by varying $\mathrm{Cu} / \mathrm{In}$ ratio

\begin{tabular}{|c|c|c|c|c|c|}
\hline $\begin{array}{c}\text { Sample } \\
(\mathrm{Cu} / \mathrm{In})\end{array}$ & $\begin{array}{c}\text { Thickness } \\
(\mu \mathrm{m})\end{array}$ & $\begin{array}{c}\mathrm{D}_{\mathrm{s}} \\
\left(\times 10^{-3} \mathrm{~cm}^{2} / \mathrm{s}\right)\end{array}$ & $\begin{array}{c}\mathrm{T}_{\mathrm{r}} \\
(\mathrm{ns})\end{array}$ & $\begin{array}{c}\mathrm{V}_{\mathrm{sr}} \\
\left(\times 10^{5} \mathrm{~cm} / \mathrm{s}\right)\end{array}$ & $\begin{array}{c}\mu \\
\left(\mathrm{cm}^{2} / \mathrm{Vs}\right)\end{array}$ \\
\hline 0.5 & 0.58 & 0.79 & 10 & $13 \times 10^{4}$ & 0.23 \\
\hline 1.0 & 0.46 & 0.85 & 350 & $2 \times 10^{3}$ & 0.80 \\
\hline 1.5 & 0.25 & 1.90 & 1 & 5 & 0.21 \\
\hline
\end{tabular}

\section{Optimization of composition of $\beta-\ln _{2} S_{3}$ thin films}

The films were irradiated with beams of energy $2.81 \mathrm{eV}$. Photothermal signal is least for the sample $2 / 8$ for (Figure 6), thereby making the non-radiative losses the least for this composition. The transport properties measured are shown in table 2 . We obtained a high mobility of $30 \mathrm{~cm}^{2} / \mathrm{Vs}$ for films prepared at $2 / 8$. But lifetime was highest for 2.5/8 in which the non- radiative (heat) loss is lower. Again lifetime was highest for 2.5/8 (1 ms). On correlating this results with $\mathrm{V}_{\mathrm{oc}}$ and $\mathrm{J}_{\mathrm{sc}}$ of CulnS $\mathrm{S}_{2} / \beta$ - $\ln _{2} \mathrm{~S}_{3}$ based cells fabricated using $\beta$ - $\ln _{2} \mathrm{~S}_{3}$ as buffer layer, we find that $\beta$ - $\ln _{2} S_{3}$ layer with $\ln / \mathrm{S}$ ratio $2 / 8$ (which offered high mobility and least $\mathrm{V}_{\mathrm{sr}}$ ) showed highest $\mathrm{J}_{\mathrm{sc}}(3.48$ $\left.\mathrm{mA} / \mathrm{cm}^{2}\right)$ and cells fabricated with $2.5 / 8$ having high life time gave maximum $\mathrm{V}_{\mathrm{oc}}(306 \mathrm{mV})$.

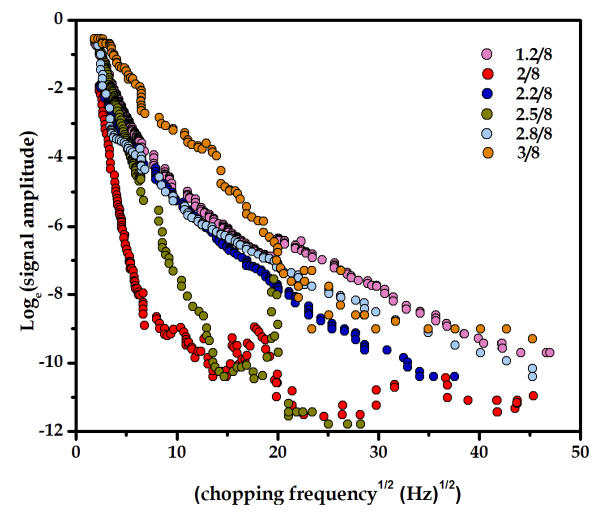

Fig 6 Photothermal signal responses for automated spray samples on $2.81 \mathrm{eV}$

Table 2 Transport properties of buffer layer

\begin{tabular}{|l|l|l|l|c|}
\hline Sample & $\begin{array}{l}\mathrm{D}_{\mathrm{s}} \\
\left(\times 10^{-3} \mathrm{~cm}^{2} / \mathrm{s}\right)\end{array}$ & $\begin{array}{l}\mathrm{V}_{\mathrm{sr}} \\
\mathrm{cm}^{2} / \mathrm{s}\end{array}$ & $\begin{array}{l}\mathrm{Tr}_{\mathrm{r}} \\
(\mu \mathrm{s})\end{array}$ & $\mu\left(\mathrm{cm}^{2} / \mathrm{Vs}\right)$ \\
\hline $1.2: 8$ & 1.2 & $2 \times 10^{8}$ & 200 & 1.46 \\
\hline $2: 8$ & 0.8 & $1 \times 10^{7}$ & 300 & 30 \\
\hline $2.2: 8$ & 0.8 & $1 \times 10^{8}$ & 40 & 0.27 \\
\hline $2.5: 8$ & 0.8 & $2 \times 10^{7}$ & 1000 & 0.5 \\
\hline $2.8: 8$ & 0.8 & $6 \times 10^{8}$ & 60 & 3.6 \\
\hline $3: 8$ & 1.1 & $1 \times 10^{8}$ & 500 & 1.9 \\
\hline
\end{tabular}


The $\mathrm{In} / \mathrm{S}$ ratio of $\beta$ - $\ln _{2} \mathrm{~S}_{3}$ buffer layer that gives high mobility, low surface recombination velocity and high lifetime has been deduced. Film 2/8 has high mobility; but the high defect density in this material reduces the lifetime of the carriers. Film with ratio, 2.5/8 with least non-radiative losses has high lifetime too. The correlation between the buffer layer transport properties and final cell parameter has been brought out from the studies, where we find that the films with high mobility and lower surface recombination velocity gave high $\mathrm{J}_{\mathrm{sc}}$ while $\mathrm{V}_{\mathrm{oc}}$ increase with increase in carrier lifetime of buffer layer.

Table 3 Electrical properties of photovoltaic cell fabricated with different $\beta$-ln ${ }_{2} S_{3}$ layers of different composition

\begin{tabular}{|c|c|c|c|}
\hline Cell & $\operatorname{In} / \mathrm{S}$ & $\begin{array}{c}\mathrm{Jsc} \\
\left(\mathrm{mA} / \mathrm{cm}^{2}\right)\end{array}$ & $\begin{array}{c}\text { Voc } \\
(\mathrm{mV})\end{array}$ \\
\hline Cell 1 & $1.2 / 8$ & 0.01 & 4 \\
\hline Cell 2 & $2 / 8$ & 3.48 & 100 \\
\hline Cell 3 & $2.5 / 8$ & 0.18 & 306 \\
\hline Cell 4 & $3 / 8$ & 0.08 & 255 \\
\hline
\end{tabular}

\section{Measurement of electrical properties of $\mathrm{CuInS}_{2} / \mathrm{ln}_{2} \mathrm{~S}_{3} / \mathrm{Ag}$ photovoltaic cell}

A CulnS $S_{2} / \mathrm{In}_{2} S_{3} / \mathrm{Ag}$ solar cell with a short circuit current density $\left(J_{\mathrm{sc}}\right)$ of $4 \mathrm{~mA} \mathrm{~cm}{ }^{-2}$ and open circuit voltage $\left(V_{\mathrm{oc}}\right) \sim$ $490 \mathrm{mV}$ is placed in the sample cell. The electrodes are connected to a load resistance, and the photothermal signal generated is measured for different values of the load resistance $\left(R_{\mathrm{L}}\right)$. The efficiency of the cell can be calculated using equation 27. Figure 7 shows a plot of the variation of the efficiency $(\gamma)$ for different values of load resistance $\left(R_{\mathrm{L}}\right)$. The data shown in Figure 7 were recorded at a modulation frequency of $15 \mathrm{~Hz}$, and the main features may be summarized as follows: it shows an optimum load resistance at around $9 \Omega$, this is the value of $R_{\mathrm{L}}$ at which $\gamma$ is maximum. For a load resistance less than $7 \Omega, \gamma$ becomes negative. It is to be noted here that $R_{\mathrm{L}}=7 \Omega$, for which $\gamma=0$ is the series resistance $\left(R_{\mathrm{S}}\right)$ of the cell. This is in agreement with the maximum power transfer theorem. Conversion efficiency as calculated using this technique is $\sim 1 \%$ which is almost the same as that determined by electrical measurements (Table 4).

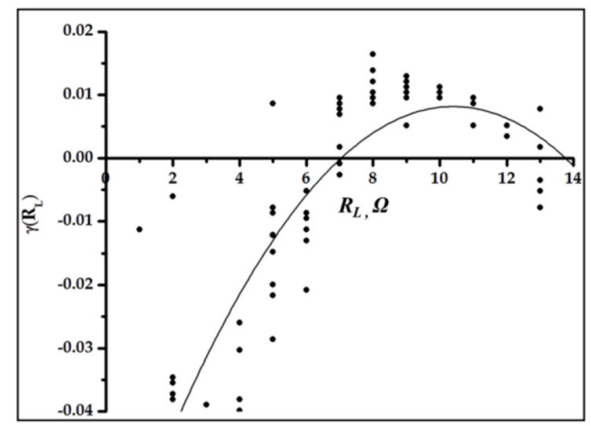

Fig 7 Photovoltaic efficiency of CulnS $S_{2} / \ln _{2} S_{3}$ solar cell with increase in load resistance

Table 4 Measured PV characteristics of CulnS $_{2} / \mathrm{In}_{2} \mathrm{~S}_{3}$ solar cell

\begin{tabular}{|l|l|l|l|}
\hline Analysis & $\begin{array}{l}R_{\mathrm{s}} \\
(\Omega)\end{array}$ & $\begin{array}{l}\text { Optimum } \\
\text { load }(\Omega)\end{array}$ & $\begin{array}{l}\text { Efficiency } \\
(\%)\end{array}$ \\
\hline Electrical & 9 & - & 1.15 \\
\hline Photothermal & 7 & 10 & 1 \\
\hline
\end{tabular}

This technique can thus be used for quality control of a solar cell in different stages of their deposition process. In the initial stages of cell fabrication, the transport parameters $\mu, V_{\mathrm{sr}}$ and $\tau_{r}$ of individual layers or during each stage of cell fabrication can be determined to check the quality of the product. Later, after cell fabrication, the series resistance, optimum load resistance, and efficiency can be measured using the photothermal beam deflection technique. 


\section{Conclusion}

A photothermal beam deflection technique is a simple and reliable tool for characterization of photovoltaic devices. This technique is employed for non-destructive measurement of the energy conversion efficiency (1\%), optimum load resistance (10 $\Omega$ ), and series resistance $(7 \Omega)$ of $\mathrm{CulnS}_{2} / \mathrm{ln}_{2} \mathrm{~S}_{3} / \mathrm{Ag}$ thin film photovoltaic cells. The study also allowed us to find out the composition best suited for device fabrication by correlating the measured transport properties of absorber and window layer with the final cell parameters The photothermal technique being a non-destructive tool and with measurements made in open circuit conditions, it can be applied to analyze partially completed solar cells, yielding information about every process in the fabrication sequence. Thus, photothermal beam deflection technique is a potential tool for industrial process monitoring. Thus, through this work, we could establish the application of photothermal technique as an efficient and simple tool for real time thin film analysis for controlling the device fabrication process.

\section{REFERENCES}

1. A G Bell, Am J Sci ., vol. 20, pp. 3051, 880

2. A G Bell, Philos Mag, vol. 11, pp. 510, 1881

3. A Rosencwaig, Review of progress in quantitative non-destructive evaluation, Plenum press, New York. 1990

4. D Fournier, C Boccara, A Skumanich, N M Amer, J Appl Phys, vol. 59, pp. 787, 1986

5. M D Dramicanin, Z D Ristowski, P M Nikolic, D G Vasiljevic, D M Todorovic Phys Rev B, vol. 51,pp. 20,1995

6. D M Todorovic, P M Nikolic, A I Bojieie, K T Radulovic, Phys Rev B, vol. 15,pp 631,1997

7. A Salinick, A Mandelis, F Funak, C Jean, Appl Phys Lett, vol. 71,pp. 1531,1997

8. A Salinick, C Jean, A Mandelis, Solid-State electron, vol. 41, pp. 591,1997

9. A Mandelis, A Othonos, C Christofides, J Bousy-Said, J Appl Phys, vol. 80, pp. 5332, 1996

10. T Ikari, A Salinick, A Mandelis, J of Appl Phys, vol. 85, pp. 7392, 1999

11. S D George, P Radhakrishnan, V P N Namboodiri, C P G Vallabhan, Phys Rev B,vol. 68,pp. 165319 2003

12. S Kumar, Appl Phys Lett, vol. 69, pp. 23, 1996

13. A F da Silva, T S da Silva, O Nakamura, M M F d’Aguiar Neto, I Pepe, L S Roman, E Veje Materials Research, vol. 4, pp. 23, 2001

14. J B Alvarado, M V Luna Anal Sci, vol.17, pp. S309, 2001

15. E Marin, I Reich, P Diaz, J J Alvarado-Gil, R Baquero, H Vargas, A Cruz-Orea, M Vargas, J Appl Phys, vol.83, pp. 2604, 1998

16. K Sakai, S Fukushima, A Fukuyama, K Usegi, I Suemune, T Ikari, IEEE Proc-Optoelectron vol. 151, pp. 328, 2004

17. M E Rodriguez, A Mandelis, G Pan, J A Garcia, Y Riopel, Anal Sci, vol.17, pp. S262, 2001

18. J Batista, A Mandelis, D Shaughnessy, Appl Phys Lett, vol. 82, pp. 4077, 2003

19. D Cahen, Appl Phys Lett, vol.33, pp. 810, 1978

20. D Cahen, S D Hale Appl Phys Lett vol.46, pp. 446, 1985

21. I F Faria, Jr C C Ghizoni, L C Miranda, H Vargas J Appl Phys vol. 59, pp. 3294, 1986

22. S I Yun, B K Yun, B K Moon, H G Seo, H L Park J Korean Phys Soc vol.18,pp. 224,1985

23. H L Riette, L C M Miranda, H Vargas Appl Phys A vol. 44, pp. 219, 1987

24. S M N Mello, C C Ghizoni, L C M Miranda, H Vargas J Appl Phys vol. 61, pp. 5176, 1987

25. P Grunow, R Schieck, J Appl Phys, vol. 77, pp. 2773, 1995

26. H Flaisher, M Wolf, D Cahen, J Appl Phys, vol. 66, pp. 1832, 1989

27. K Boubaker, Sol Energy Mater Sol Cells, vol. 91, pp. 1319, 2007

28. A C Boccara, D Fournier, W Jackson, N M Amer, Opt Lett ,vol. 5 , pp. 377, 1980

29. J A Sell, D M Heffelfinger, P L G Ventzek, R M Gilenbach, J Appl Phys vol. 3, pp. 69, 1981

30. M Bertolotti, L Fabbri, C Sibilia, A Ferrari, N Sparvier, G Suber, J Phys D: Appl Phys, vol. 21, pp. S14, 1988

31. S D George, P Radhakrishnan, V P N Nampoori, C P G Vallabhan, Phys Rev B vol.68, pp. 1653192003

32. A Rosencwaig, Opt Comm, vol. 7, pp. 305, 1973

33. U Zammit, F Gasparrini, M Marinelli, R Pizzoferrato, F Scuderi, S Martellucci, J Appl Phys vol. 69, pp. 2577, 1991

34. D Fournier, C Boccara, A Skumanich, N M Amer, J Appl Phys vol. 59, pp. 787, 1986

35. H L Riette, L C M Miranda, H Vargas, Appl Phys A, vol. 44, pp. 219, 1987

36. Y Akaki, K Nomoto, S Nakamura, T Yoshitake, K Yoshino, J Phys: Conf Ser vol.100, pp. 082022, 2008

37. K Dejessas, G Masse, Ibannaim, J of Electrochem Soc, vol. 147, pp. 1235, 2000 